\title{
Weighted Reconstruction and Improved Eigenclass Combination Method for the Detection of Bearing Faults
}

\author{
Zhengyu Du, ${ }^{1}$ Jie Ma ${ }^{1},{ }^{1}$ Chao $\mathrm{Ma}^{2}{ }^{2}$ Min Huang, ${ }^{1}$ and Weiwei Sun ${ }^{1}$ \\ ${ }^{1}$ School of School of Mechatronics Engineering, Beijing Information Science and Technology University, Beijing, China \\ ${ }^{2}$ Key Laboratory of Modern Measurement and Control, Ministry of Education, Beijing, China \\ Correspondence should be addressed to Jie Ma; mjbeijing@163.com
}

Received 4 August 2021; Accepted 26 October 2021; Published 10 November 2021

Academic Editor: Yong Chen

Copyright (c) 2021 Zhengyu Du et al. This is an open access article distributed under the Creative Commons Attribution License, which permits unrestricted use, distribution, and reproduction in any medium, provided the original work is properly cited.

Aiming at the difficulty of extracting and classifying early bearing faults, a fault diagnosis method based on weighted average timevarying filtering empirical mode decomposition and improved eigenclass is proposed in this paper. Firstly, the bearing fault signal is decomposed into a series of intrinsic mode functions by the signal decomposition method, and the amplitude of the component is modulated by the weighted average method to enhance the fault impulse component. Then, the fractional Fourier transform is used to filter the reconstructed signal. Regarding classification issues, the eigenclass classifier is optimized by the IDE method that can be used for feature dimensionality reduction. Finally, the optimal features are selected and input into the IDE-EigenClass model. The experimental results show that the bearing fault diagnosis method proposed in this paper has higher accuracy and stability than the traditional PNN, SVM, BP, and other methods.

\section{Introduction}

In the process of bearing fault diagnosis, one difficult point is to extract the fault impulse component. Because the background noise is present in the process of equipment operation, the fault impulse component is likely to be drowned by the noise, resulting in the failure of diagnosis [1-3]. To solve this problem, Huang et al. proposed the empirical mode decomposition (EMD) for the first time, which can effectively reduce the noise in the signal. However, EMD has some problems such as end effect and mode aliasing [4]. Based on EMD, Huang et al. proposed the ensemble empirical mode decomposition (EEMD). By adding noise to the EMD decomposition process, the effect of mode aliasing has been significantly reduced [5]. In 2014, the variational mode decomposition (VMD) was proposed. VMD takes into full consideration the narrow-band property of the components, so the filtering frequency band is more centralized and the signal-to-noise ratio of the signal components is higher [6]. However, there remains the problem of mode aliasing. The time-varying filtering empirical mode decomposition (TVFEMD) is a new signal decomposition method proposed by Li et al. in 2017 [7]. The method provides an effective solution to the above problems by using B-spline approximation as a time-varying filter.

In addition, the noise can be removed by filtering the signal, as by the method of combining fast spectral kurtosis with VMD to successfully extract the features of early rolling bearing faults [8]. On the basis of the fast spectral kurtosis method, autocorrelation spectral kurtosis has been improved to effectively eliminate the interference of nonperiodic noise and improve the recognition accuracy [9]. In recent years, the fractional Fourier transform (FRFT) has been applied intensively in fault diagnosis. The fractional Fourier transform has good time-frequency focusing property for LFM signals [10]. In the early stage of the fault, since the fault impulse frequency changes steadily, the fractional Fourier transform proves effective in extracting the fault impulse components. Jiao and Ma used FRFT to extract the features of the bearing signal, and the effect was significant [11]. In addition, the advantages of FRFT can be exploited to the full under variable conditions. Jia and Huang [12] successfully diagnosed the fault of variable-speed bearing using FRFT and LMS noise reduction methods. 
Machine learning algorithms are used mainly for classification issue. For example, Zhang et al. used the EMDSVM combination method to classify faults [13]. In addition, Jung and Koh used the DWT-KNN to classify faults and achieved certain results [14]. However, there remain many problems in classification. For example, the accuracy is not high enough, and the operation is too time-consuming. Standardized variable distance (SVD) is an improved algorithm based on MDE and proposed in 2020 to solve the problem of long operation time [15]. However, its classification accuracy is heavily dependent on features. In the neural network algorithm, Ma et al. used the CSBP method to improve the fault recognition rate by optimizing the BP neural network [16]. In addition, the convolutional neural network (CNN) [17] has also been used to classify the bearing faults. Eigenclass is a new machine learning classification method proposed by Uğur in 2020. In this procedure, a feature matrix is constructed before the proximity based on the eigenvalues and is used to classify the samples [18]. This method ensures not only a high accuracy but also a high speed and efficiency when the sample size is not very large.

The above studies mainly focus on the vibration signal. Nevertheless, for some devices which are not easily embedded with the acceleration sensor, it would be a good choice to use the acoustic pressure sensor to collect the acoustic signal for analysis and processing. Its advantage of noncontact measurement makes it possible to diagnose the fault of some devices in which it would otherwise be difficult or even infeasible to embed the acceleration sensor [19]. However, there are few studies on fault diagnosis methods based on acoustic signals probably because of the relatively low signal-to-noise ratio of the acoustic signal. In addition, the generated noise still contains certain impulse noise, which is not necessarily periodic but can have a great effect on the kurtosis of the signal. Therefore, the impulse noise may lead to the wrong selection of sensitive components.

To solve the above problems, a fault diagnosis method based on weighted average time-varying filtering empirical mode decomposition and improved eigenclass is proposed in this paper. Firstly, the acoustic array sensor is used to collect data. Then, the signal is preprocessed to reduce noise interference. Finally, the features are input into IDEEigenclass model for fault classification. Compared with the existing bearing fault diagnosis methods, the innovative point of this proposal is that the acoustic array sensor is used to collect the bearing fault signal. This method aims to realize the multichannel acquisition, which can not only improve the accuracy of bearing sound signal but also realize the localization of the sound source of fault bearing. In the process of actual equipment operation, there are usually more than one bearing, usually multiple or even multiple groups of bearings. In such a scenario, failure to accurately locate whichever of the bearings will make it inconvenient to maintain the equipment. The acoustic array technology has made it possible to accurately locate the pitch angle and azimuth of the bearing as well as the faulty bearing. This paper is organized as follows. Section 2 mainly introduces the principle of signal preprocessing. Section 3 introduces the principle of classification for optimization of IDEEigenclass. Section 4 deals with the diagnosis process. Section 5 is concerned on the experimental verification. Finally, a summary with relevant discussion is made in Section 6.

\section{Signal Preprocessing}

2.1. Time-Varying Filtering Empirical Mode Decomposition. Time-varying filtering empirical mode decomposition (TVFEMD) is an improved method based on EMD. Because the cut-off frequency in those methods based on filtering is constant over time, they are not suitable for nonstationary signals. This method can solve the problem of mode mixing while maintaining the time-varying characteristics of different modes. The detailed introduction on TVFEMD has been covered in [20] and will not be repeated here.

2.2. Signal Reconstruction. A new signal reconstruction method is proposed in this paper.

Firstly, the kurtosis of each component is calculated. The kurtosis can reflect the degree of a signal's transient state, thereby identifying the fault impulse characteristics. The expression is given as follows:

$$
k=\frac{(N-1) \sum_{i=1}^{N}\left(x_{i}-\bar{x}\right)^{4}}{\left(\sum_{i=1}^{N}\left(x_{i}-\bar{x}\right)^{2}\right)^{2}} .
$$

Next, the coefficient of correlation between each component and the original signal is calculated, with the coefficient of correlation between two signals defined as

$$
\rho_{x y}=\frac{\sum_{i=1}^{N}\left(X_{i}-\bar{X}\right)\left(Y_{i}-\bar{Y}\right)}{\sqrt{\sum_{i=1}^{N}\left(X_{i}-\bar{X}\right)^{2}} \sqrt{\sum_{i=1}^{N}\left(Y_{i}-\bar{Y}\right)^{2}}},
$$

where $\bar{X}$ and $\bar{Y}$ are the mean values of $X$ and $Y$, respectively, and $N$ is the size of the dataset.

The product of kurtosis and coefficient of correlation is termed as correlation kurtosis:

$$
s(i)=k(i) \times \rho(i) .
$$

The mean correlation kurtosis is calculated as

$$
S(i)=\frac{s(i)}{\sum_{i=1}^{N} s(i)},
$$

where $s(i)$ represents the correlation-kurtosis value of each IMF component.

The signal is weighted and reconstructed by

$$
\mathrm{IMF}_{K}^{i}=\sum_{i=1}^{N} S(i) \mathrm{IMF}^{i}
$$

The above method is the so-called weighted average time-varying filtering empirical mode decomposition.

This method is tantamount to modulating the amplitude at different frequency components of the signal. The kurtosis correlation coefficient index is proposed to modulate the amplitude of each component. By multiplying the index 
factor by each component, the effective component can be enhanced, while the noise component can be reduced in amplitude, thereby pinpointing the fault component. At the same time, this method retains all IMF components to ensure the intactness of useful information, thereby improving the accuracy of diagnosis.

2.3. Fractional Fourier Transform. The fractional Fourier transform is based on the Fourier transform. Its physical significance is shown in Figure 1.

As shown in Figure 1, a signal is transformed from the time domain into the frequency domain through the Fourier transform, a process equivalent to rotating the signal by $90^{\circ}$. In other words, the physical significance of the fractional Fourier transform is equivalent to the whole transformation process of the signal at $0^{\circ}$ to $90^{\circ}$. The Fourier transform is applied to every internal angle to find the optimal rotary angle through two-dimensional peak search. The principle of selection for the optimal rotary angle is described as follows. The rotary angle is optimal when the projection of the desired fault impulse component on the optimal plane of rotation does not interfere with the projection of the noise. Since the projection of noise at any rotary angle is invariable, the only consideration is the projection of the fault impulse component.

\section{Feature Optimization and Fault Classification}

3.1. IDE Feature Optimization. In recent years, machine learning has been applied more and more frequently in the research on classification problems. Two key factors that affect the effectiveness of classification are the quality of feature selection and the classification method used. The foremost step of classification is to select features, whereby to use the corresponding features to represent a section of data. The quality and the number of features can have a great impact on the effectiveness of classification. In addition, the number of features should be moderate. Too few features are not enough to make a distinction between the types of samples, while too many features may lead to the disaster of dimensionality, resulting in a sharp decline in classification accuracy. Overemphasizing the distinction between features may lead to overtraining, which means training the wrong features in the original data as correct ones at certain probability. Anyway, either an extreme number of features or overemphasis on the distinction between features may lead to a sharp decline in classification accuracy.

The improved distance evaluation (IDE) technique is a feature optimization method. The ratio of the average distance between the same type of samples to the average distance between different types of samples is calculated to judge whether the feature can make a distinction between the types [21]. All features are solved and normalized according to the above method. The numerical results are sorted, and the optimal features are selected by setting a certain threshold. For example, a feature set contains class $B$ samples, and its expression is given as follows:

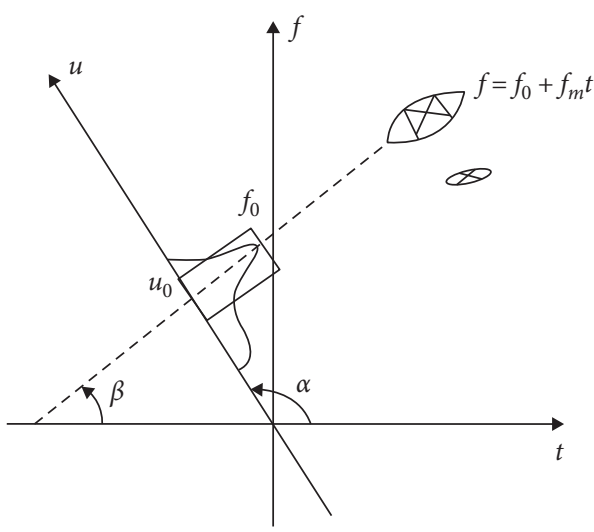

FIgURE 1: The physical significance of FRFT.

$$
\left\{p_{n, b, j}, n=1,2, \ldots N_{b} ; b=1,2, \ldots, B ; j=1,2, \ldots, J\right\}
$$

where $p_{n, b, j}$ represents the $j^{\text {th }}$ eigenvalue of the $n^{\text {th }}$ sample in the class $B$ sample, $N_{b}$ represents the sample size of class $B, J$ represents the eigenvalue, and $B$ stands for the class. Therefore, the distinguishing degree of a feature to all classes can be calculated by the mathematical formula.

First, the average distance between the eigenvalues of the same samples is calculated as

$$
\begin{aligned}
d_{b, j} & =\frac{1}{N_{b} \times\left(N_{b}-1\right)} \sum_{l, n=1}^{N_{b}}\left|p_{n, b, j}-p_{l, b, j}\right|, \\
l, b & =1,2, \ldots, N_{b}, l \neq m .
\end{aligned}
$$

Then, we calculate the average value $d_{j}^{(w)}$ :

$$
d_{j}^{(w)}=\frac{1}{B} \sum_{b=1}^{B} d_{b, j} .
$$

Define the variance factor of $v_{j}^{(w)}$ as

$$
v_{j}^{(w)}=\frac{\max \left(d_{b, j}\right)}{\min \left(d_{b, j}\right)} .
$$

Calculate the mean value of all samples under the same condition:

$$
u_{b, j}=\frac{1}{N_{b}} \sum_{n=1}^{N_{b}} q_{n, c, j} .
$$

Calculate the average distance between different samples:

$$
\begin{aligned}
d_{j}^{(b)} & =\frac{1}{B \times(B-1)} \sum_{b, e=1}^{B}\left|u_{e, j}-u_{b, j}\right|, \\
b, e & =1,2, \ldots, B, b \neq e .
\end{aligned}
$$

Define the variance factor $v_{j}^{(b)}$ as

$$
v_{j}^{(b)}=\frac{\max \left(\left|u_{e, j}-u_{b, j}\right|\right)}{\min \left(\left|u_{e, j}-u_{b, j}\right|\right)}, \quad b, e=1,2, \ldots, B, b \neq e .
$$

A compensation coefficient $\lambda$ is introduced as 


$$
\lambda_{j}=\frac{1}{\left(v_{j}^{(w)} /\left(\max \left(v_{j}^{(w)}\right)\right)\right)+\left(v_{j}^{(b)} /\left(\max \left(v_{j}^{(b)}\right)\right)\right)} .
$$

The ratio between the two average distances is calculated and the compensation coefficient is allocated:

$$
\alpha_{j}=\lambda d_{j} \frac{d_{j}^{(b)}}{d_{j}^{(w)}} .
$$

Finally, $\overline{\alpha_{j}}$ is normalized as

$$
\overline{\alpha_{j}}=\frac{\alpha_{j}}{\max \left(\alpha_{j}\right)}
$$

After normalization, the features are sorted in the size order, with the optimal features selected according to the threshold to form a feature matrix, which is then input into the machine learning model for training.

3.2. Eigenclass. According to the method, the feature matrix is first made up out of each set of features; next, the eigenvalues of the feature matrix in the training set and the test set are determined; finally, the distance between them is calculated.

Step 1: divide the dataset $A$ into the matrix of the training set $A_{\text {train }}$ and the test set $A_{\text {test }}$.

Step 2: if 0 is an element of the matrix, replace it with a very small positive number, such as 0.0001 .

Step 3: for each class $r$, divide the training set into the training matrix $A_{\text {train }}^{r}$ according to the number of classes.

Step 4: each line element of the test set is composed of the feature matrix, so is each line element of each class in the training set. Then, the eigenvalues of the test matrix and all the training matrices are obtained by

$$
b_{r t}^{i}=q\left(\operatorname{diag}\left(A_{t-\text { train }}^{r}\right), \operatorname{diag}\left(A_{i-\text { test }}\right)\right) .
$$

Step 5: calculate the quasi-distance between the training matrix and the test matrix by the following definition:

$$
q(A, B)=\sum \|\left[\begin{array}{l}
1 \\
\cdot \\
\cdot \\
1
\end{array}\right]-\operatorname{eig}(A, B) \mid
$$

Step 6: derive the quasi-distance between the test set and all the training sets of the same type, and then, construct a distance matrix and reorganize it in the ascending order.

Step 7: calculate the $r$-mean value of each line element. The test set of the line with the minimum mean value belongs to the class represented by this line.

The steps of the eigenclass algorithm are shown in Table 1.

\section{Procedure}

According to the method proposed in this paper, a flowchart can be drawn as shown in Figure 2. The diagnostic steps in this paper are shown in Table 2.

\section{Experimental Verification}

5.1. Feature Extraction. To further verify the effectiveness of the method proposed in this paper, the NU1004 cylindrical roller bearing is used to test the outer ring fault, with bearing parameters shown in Table 3 . The rolling bearing fault testbed is shown in Figure 3. The test platform consists of a rolling bearing, a DC motor, a support bench, a loading bolt, and other components. The speed of the bearing is adjusted by the motor, and the loading bolt is used to load the bearing. The test speed has reached $780 \mathrm{rpm}$. ReSpeaker Mic Array v2.0 is used as an acoustic sensor. The sampling frequency is $44.1 \mathrm{kHz}$. The device is shown in Figure 4. Placed at a horizontal distance of $20 \mathrm{~cm}$ from the bearing, the sensor can implement 4-channel acquisition. Two arrays of such sensors are placed in the axial and radial directions of the bearing, respectively, to implement a total of eight channels of acquisition.

According to the main parameters of the bearing, the theoretical fault characteristic frequency is $64.48 \mathrm{~Hz}$ at a test speed of $780 \mathrm{rpm}$. The collected sound signal is decomposed by TVFEMD to obtain 15 intrinsic mode functions. The time-domain waveform of the original signal and some IMF components are shown in Figure 5. The purpose of selecting components $6,7,12$, and 13 is that the kurtosis correlation coefficient index of the four components exceeds the threshold value of 0.7 after normalization, an indication that these components are most sensitive. The kurtosis and the coefficient of correlation are calculated as shown in Figure 6.

Figure 5 indicates that IMF component 1 has a relatively large kurtosis value. By the kurtosis selection principle, it should contain the richest fault information. However, the envelope spectrum shows that it has a peak value near $100 \mathrm{~Hz}$, which is quite discrepant from the theoretical fault characteristic frequency of $64.48 \mathrm{~Hz}$. The discrepancy may be ascribed to the impulse noise contained in this component rather than in the fault impulse component. By calculating the coefficient of correlation, it is found that the correlation with the original signal is very low, and therefore, it can be concluded that IMF 1 is not an effective component.

By multiplying the kurtosis correlation coefficient index by the amplitude of each IMF, the amplitude of each component is modulated to increase the ratio of sensitive component to noise component, thus fulfilling the purpose of feature enhancement.

Then, the reconstructed signal is filtered by undergoing the fractional Fourier transform, and the result is obtained at $90^{\circ}$ to $180^{\circ}$, where the optimal rotary angle is found, as shown in Figure 7, and the best order is 1.01 .

At the order of 1.01, narrow-band filtering is carried out, with the results shown in Figure 8.

Then, the inverse fractional Fourier transform is performed, with the results shown in Figure 9. 
TABLE 1: Steps for eigenclass classification.

Eigenclass algorithm

Step 1: divide the data $A$ into the matrix of the training set $A_{\text {train }}$ and the test set $A_{\text {test }}$

Step 2: if $\begin{aligned} & a_{i j \text {-train }}=0, a_{i j \text {-train }} \longleftarrow 0.0001 \\ & a_{i j \text {-test }}=0, a_{i j \text {-test }} \longleftarrow 0.0001\end{aligned}$

Step 3: for each class $r$, extract the $r$-class training matrix $A_{\text {train }}^{r}$ from $A_{\text {train }}$

Step 4: for each $i$, obtain the eigenvalues of the test matrix and all the training matrices:

$b_{r t}^{i}=q\left(\operatorname{diag}\left(A_{t-\text { train }}^{r}\right), \operatorname{diag}\left(A_{i-\text { test }}\right)\right)$
Step 5: calculate the quasi-distance between the training matrix and the test matrix by the following definition:q $q(A, B)=\sum\left|\left[\begin{array}{c}1 \\ \vdots \\ 1\end{array}\right]-\operatorname{eig}(A, B)\right|$
Step 6: construct a distance matrix $C$ and reorganize it in ascending order

Step 6: construct a distance matrix $C$ and reorganize it in ascending order

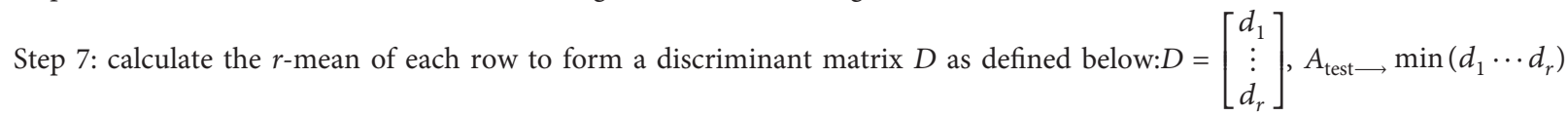

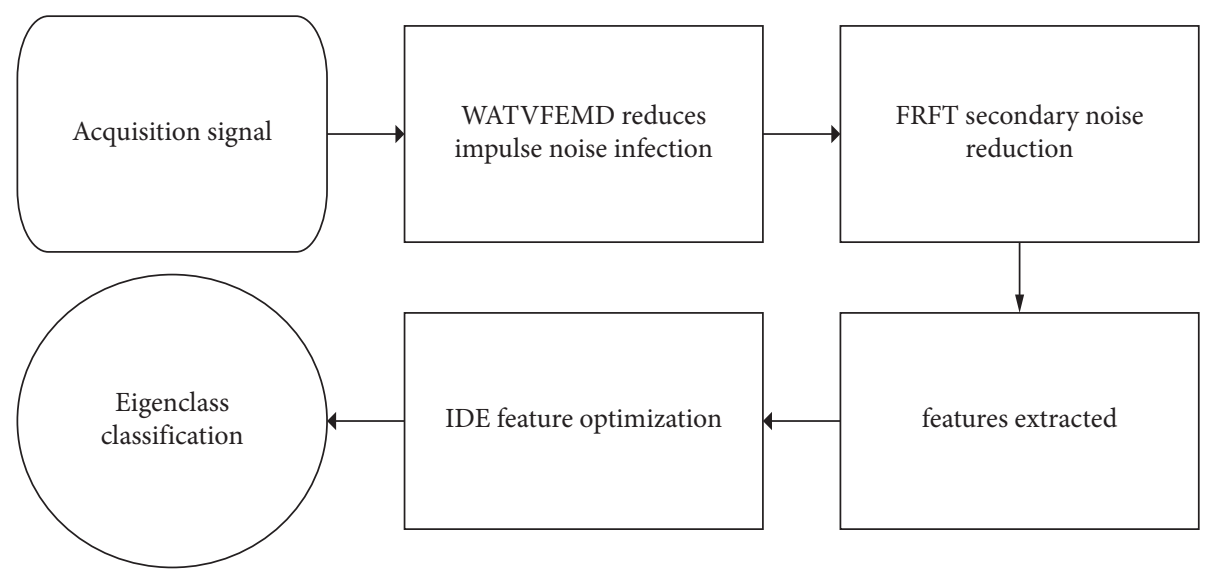

FIgURe 2: A flowchart of fault diagnosis.

TABLe 2: Fault diagnosis procedure.

Diagnostic procedure

Step 1: decompose the original signal by TVFEMD to extract the IMFs component

Step 2: obtain the correlation-kurtosis index of each modal function and weight and reconstruct the signal

Step 3: use an FRFT filter for secondary noise reduction to improve the signal-to-noise ratio

Step 4: extract 18 feature indexes including time domain and entropy, and then, use the IDE for feature selection to form a feature set

Step 5: divide the data into the training set and the test set and combine them into the eigenclass for classification prediction

TABle 3: Main parameters of the NU1004 bearing.

\begin{tabular}{lcccc}
\hline Internal diameter $(\mathrm{mm})$ & External diameter $(\mathrm{mm})$ & Pitch diameter $(\mathrm{mm})$ & Diameter of element $(\mathrm{mm})$ & Number of balls \\
\hline 20 & 42 & 31 & 5.5 & 12 \\
\hline
\end{tabular}

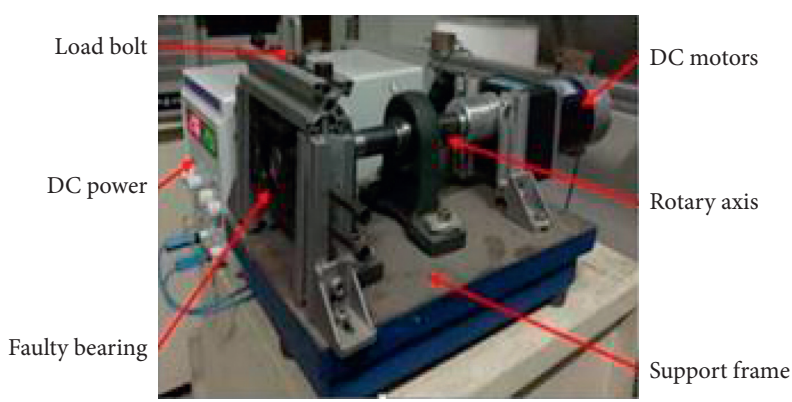

FIGURE 3: Bearing test platform. 


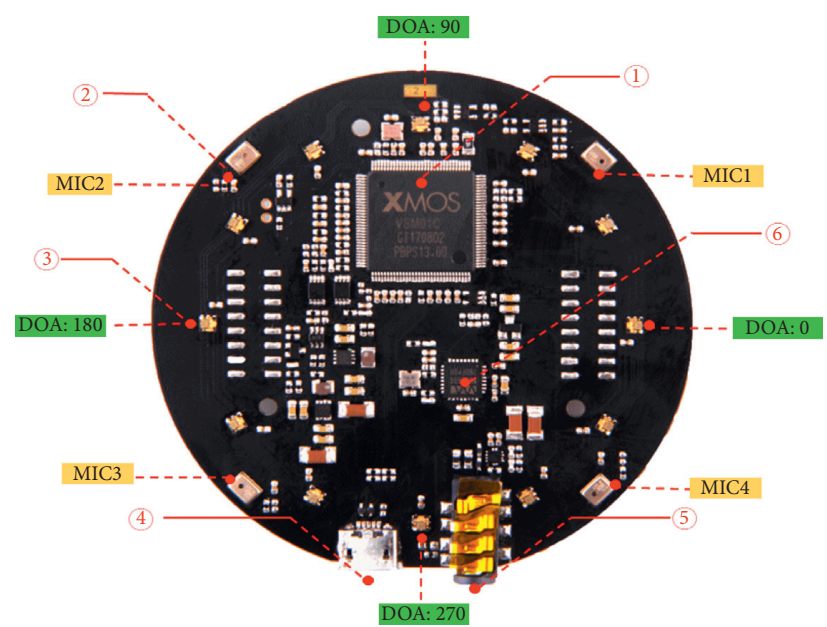

FIgURE 4: Schematic view of the sensor.
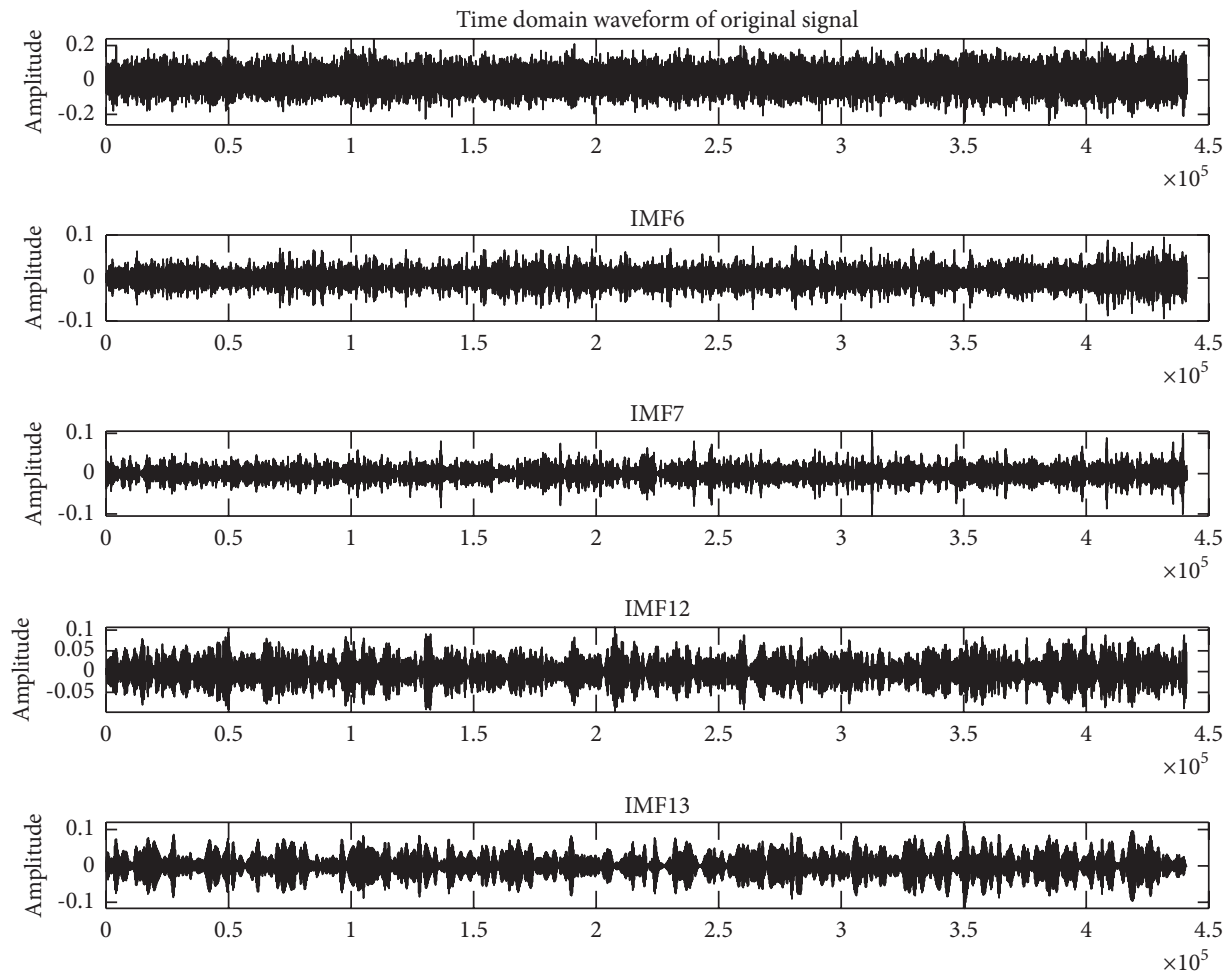

FIGURE 5: Time-domain waveforms of the original signal and some IMF components.

Figure 9 indicates that the fault characteristic frequency is $64.66 \mathrm{~Hz}$. Given its bandwidth of $1 \mathrm{~Hz}$, its harmonics and other noise components are filtered out. Therefore, the envelope spectrum contains only the fault characteristic frequency. It follows that the proposed method proves effective.

5.2. Fault Classification. The fault data of normal bearing, inner ring bearing, outer ring bearing, and rolling element bearing have been collected. More details of the dataset is presented in Table 4.
After all data are denoised, 15 time-domain indexes and 3 entropy indexes are selected to represent a segment of data. The time-domain characteristics are shown in Table 5. Three entropy indexes, namely, sample entropy, permutation entropy, and fuzzy entropy, have also been selected.

Then, the IDE is used for feature optimization, and the result is shown in Figure 10.

The threshold is set to 0.6. As shown in Figure 10, the threshold values of features 12, 13, 15, and 17 are greater than 0.6 , an indication that these four features can make a good distinction between the experimental data. 

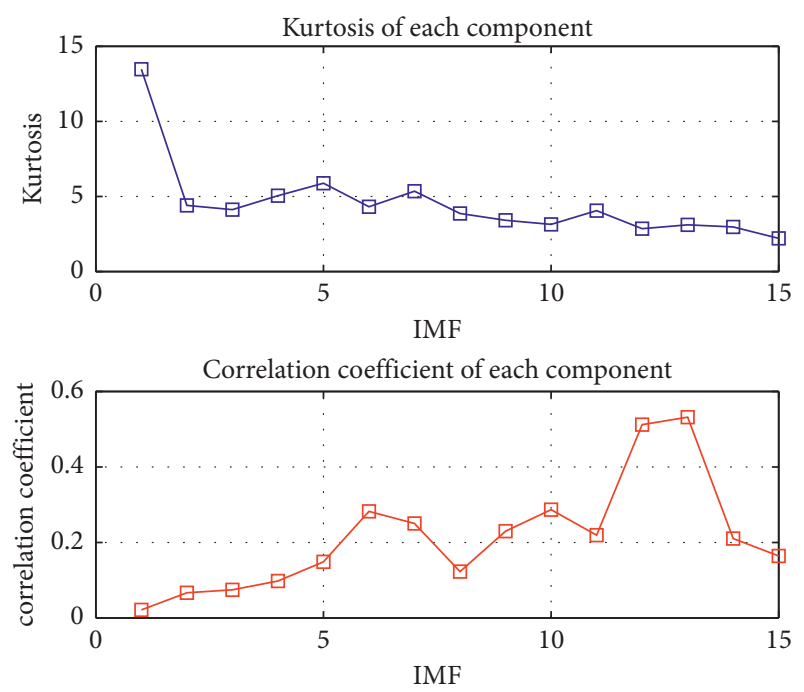

FIgURE 6: Kurtosis and correlation coefficient of each component.

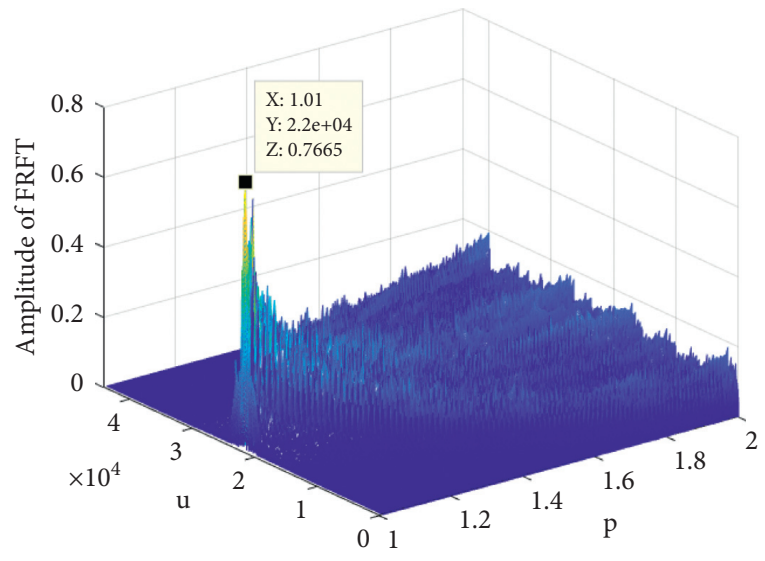

FIgURE 7: FRFT diagram.

Then, 400 datasets are combined according to the ratio of $1: 4$ to form the training set and the test set. Finally, the eigenclass is used to classify the data. The results are shown in Figure 11.

Figure 11 indicates that the eigenclass has a satisfactory effect of classification on the test set, with only 3 label classification errors, including only one classification error for normal bearing, inner circle, and outer ring, respectively. The classification accuracy of $96.25 \%$ demonstrates that the eigenclass has a good effect on bearing fault classification. The effectiveness of this method is preliminarily verified.

In addition, this paper makes the comparison and verification in two aspects. Firstly, to verify whether the eigenclass has sufficiently accurate classification effect, the processed data are input into PSO-SVM, BP, PNN, and SVD. 10 training sessions are conducted, respectively, with the results of one testing shown in Figure 12. The comparison of testing accuracy for 10 times is shown in Figure 13.

As can be seen from Figure 12, the eigenclass achieves the highest testing accuracy within the least operation time. Therefore, it can be verified that eigenclass has a better classification effect than the traditional classification methods.
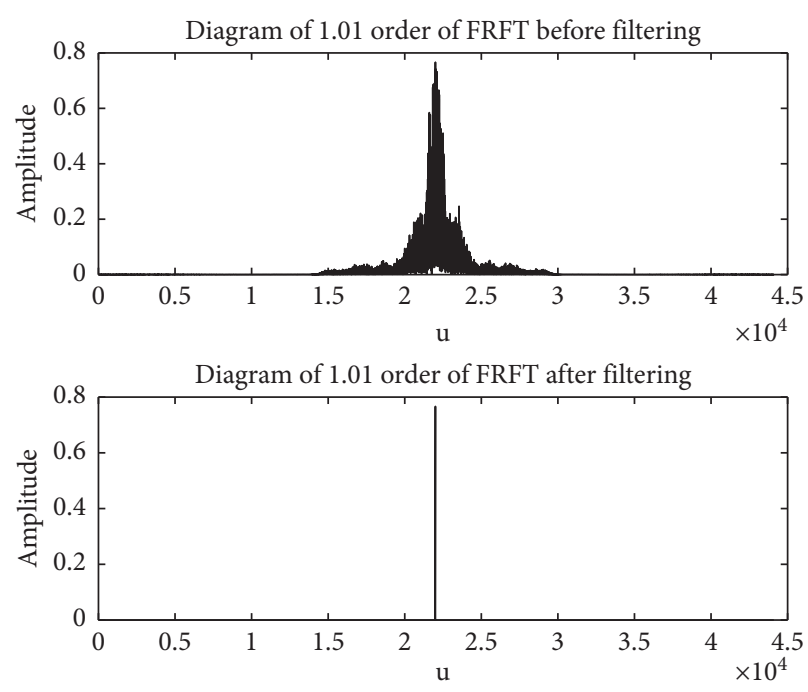

FIGURE 8: Fractional Fourier transform filter.
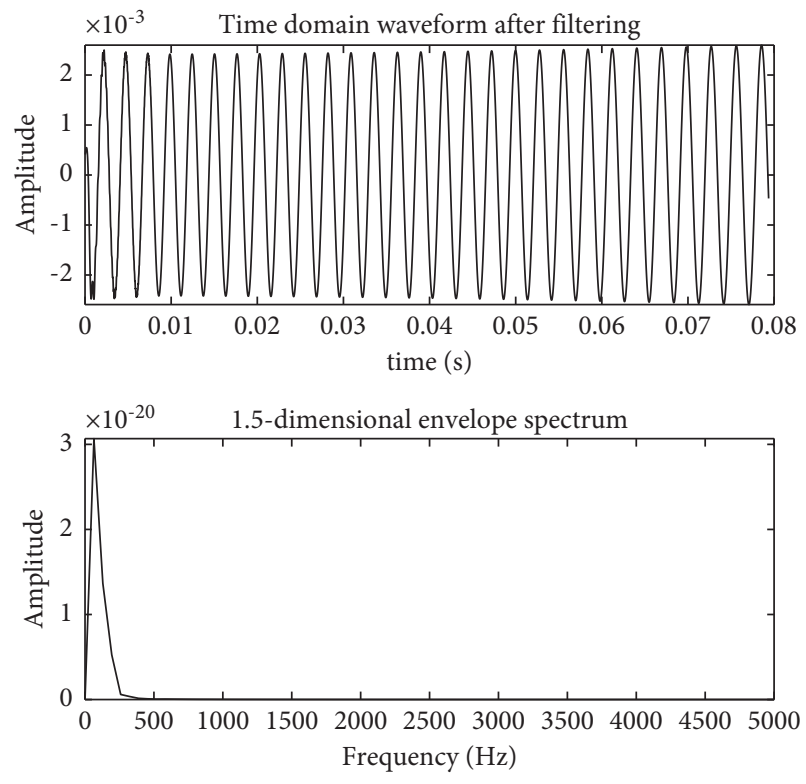

FIgURE 9: Partial time-domain waveform after filtering.

Table 4: Details of the bearing dataset.

\begin{tabular}{lccc}
\hline Training samples & Testing samples & Operating condition & Label \\
\hline 80 & 20 & Normal & 1 \\
80 & 20 & Inner race & 2 \\
80 & 20 & Out race & 3 \\
80 & 20 & Ball & 4 \\
\hline
\end{tabular}

Secondly, to verify the effectiveness of the IDE method and the robustness of the eigenclass method, four features are randomly selected and input into the above five classification models, with the results shown in Table 6 .

Table 4 indicates that the IDE method can improve the success rate of classification. In addition, no matter which four features are used, the eigenclass achieves the highest classification accuracy. Thus, the effectiveness of the proposed method is verified. 
TABLE 5: Feature parameters.
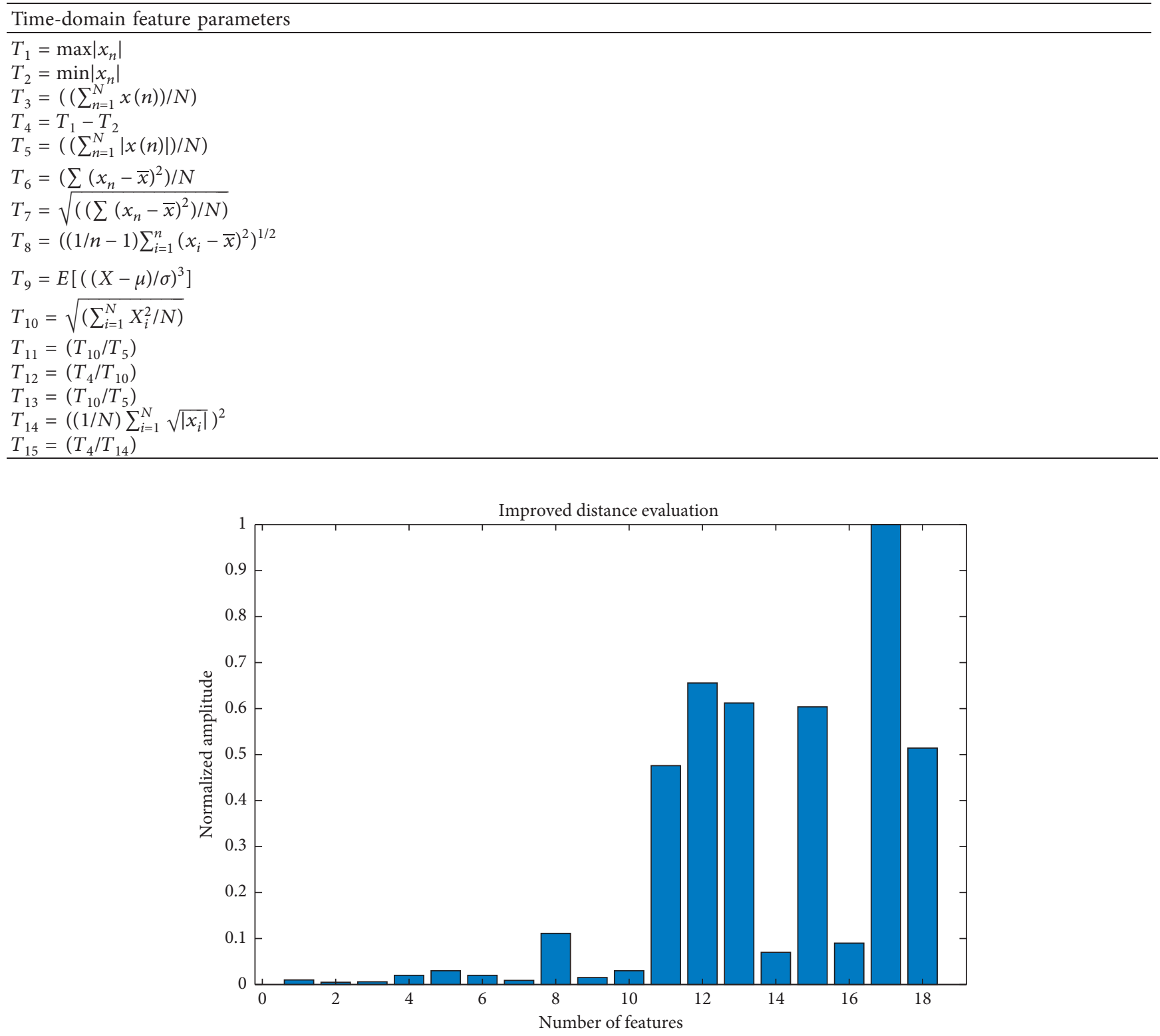

Figure 10: IDE feature optimization.
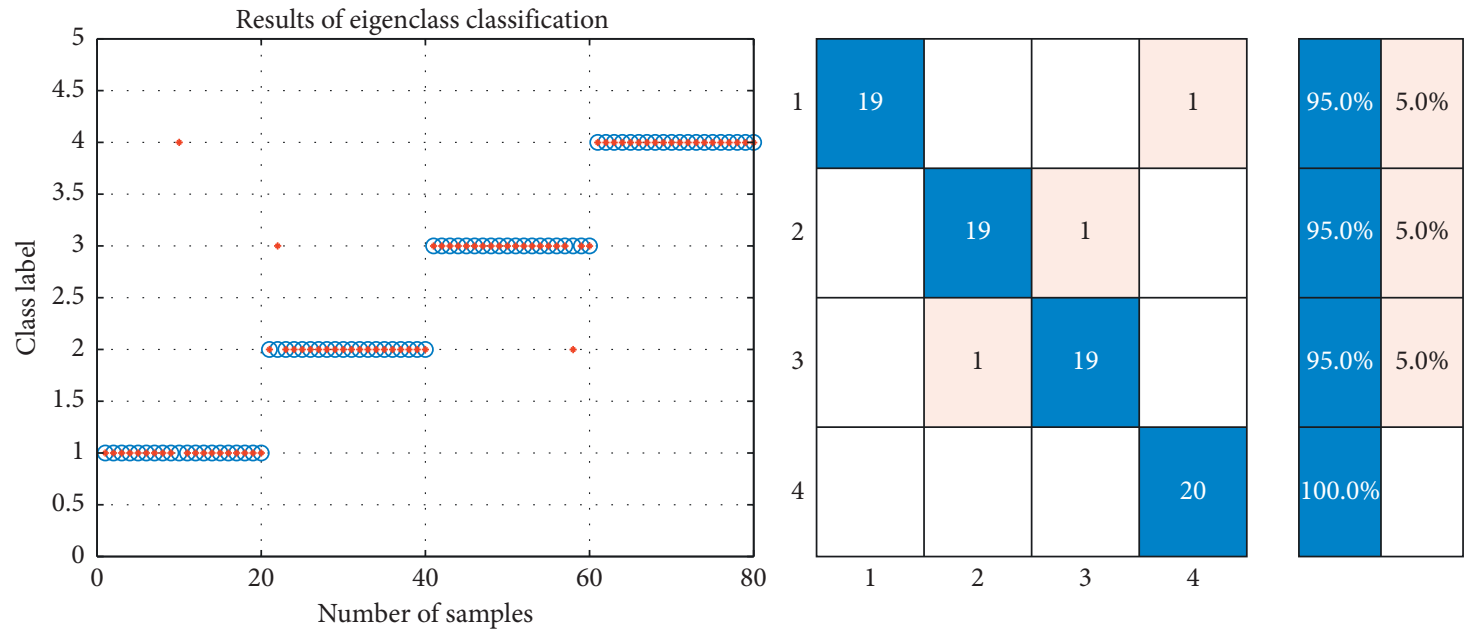

Figure 11: Classification results. 

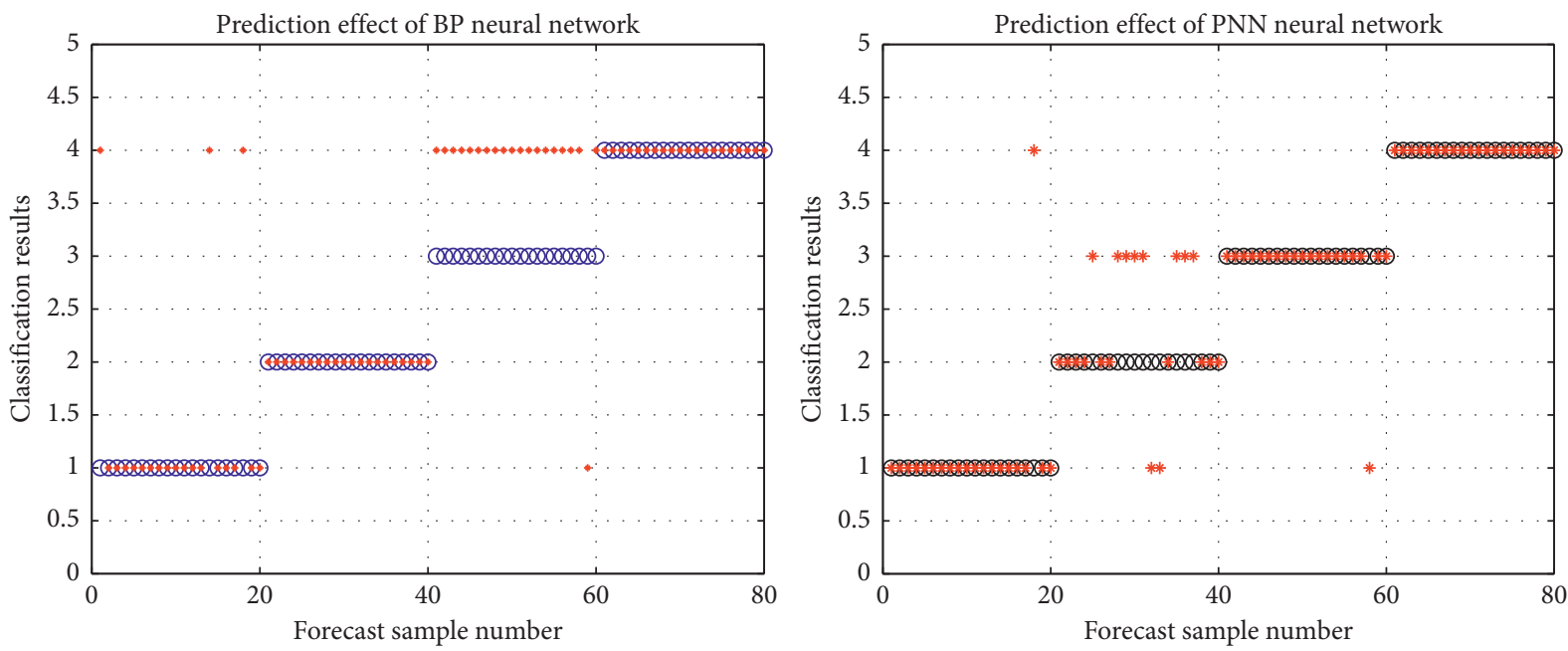

- Actual value

- Predicted value

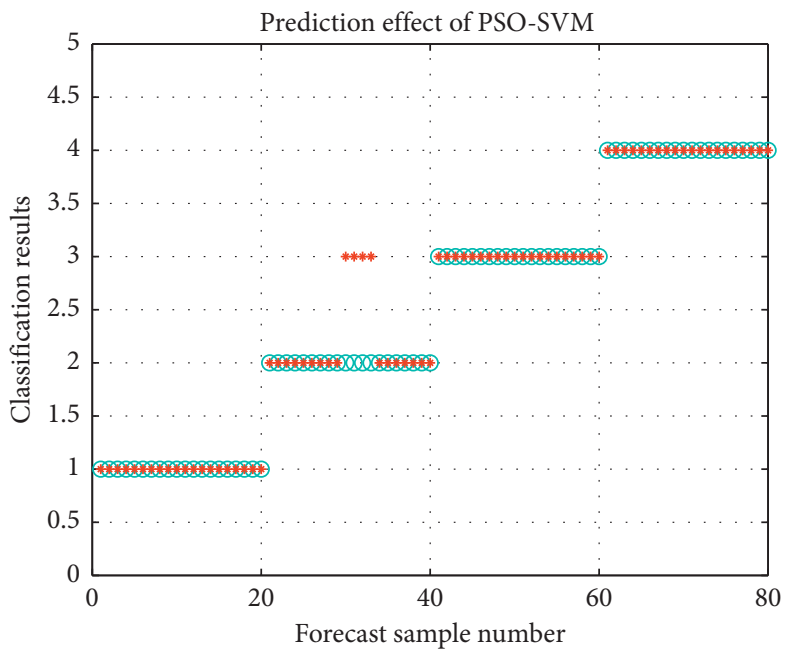

- Actual value

- Predicted value

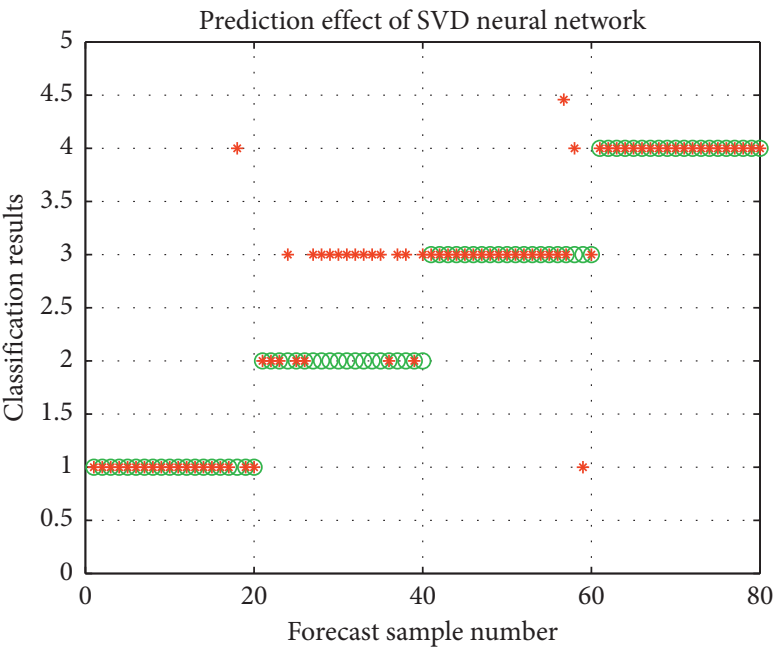

- Actual value

- Predicted value

- Actual value

- Predicted value

FIgURE 12: Comparison of classification results of different methods.

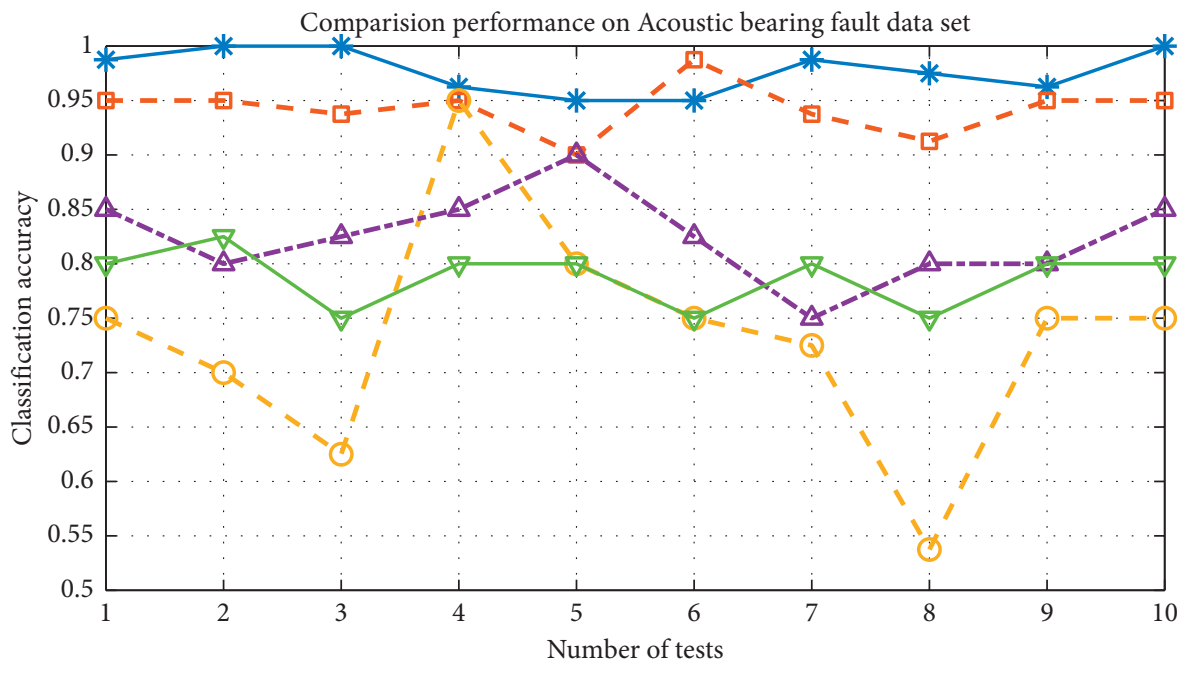
* Eigenclass
-日- PSO-SVM
-A- PNN
$-\ominus-$ BP
$-\nabla-$ SVD

FIgURE 13: Classification accuracy of different methods for 10 times. 
Table 6: Performance comparison for different features.

\begin{tabular}{lcccc}
\hline Accuracy & & \multicolumn{3}{c}{$\begin{array}{c}\text { Four features selected } \\
\text { randomly }\end{array}$} \\
& Superior features & Min & Mean & Max \\
\hline Eigenclass (\%) & 100 & 75.00 & 87.50 & 100 \\
PSO-SVM (\%) & 97.50 & 62.50 & 78.75 & 95.00 \\
BP (\%) & 95.00 & 50.00 & 72.50 & 95.00 \\
PNN (\%) & 90.00 & 65.00 & 77.50 & 90.00 \\
SVD (\%) & 82.50 & 60.00 & 71.25 & 82.50 \\
\hline
\end{tabular}

\section{Conclusion}

In this paper, a bearing fault diagnosis method has been proposed, based on weighted average time-varying filter modal decomposition and improved eigenclass. Through experimental verification, the following conclusions can be drawn:

(1) The method of weighted reconstruction of decomposed signal proposed in this paper can effectively modulate the amplitude of signal, increase the ratio of the fault impulse component to the noise component, and improve the accuracy of classification.

(2) By optimizing the input of the classifier, the classification accuracy of the eigenclass has been improved with effectiveness. Compared with traditional methods, this novel method ensures both the highest accuracy and stability, as well as the verified effectiveness.

(3) In this paper, the eigenvalue attributes between indexes are used to construct the eigenvalue matrix. Concerning its originality, this method is innovative and open to further exploration and improvement in the follow-up research.

\section{Data Availability}

The data of this paper can only be obtained with the consent of the corresponding author.

\section{Conflicts of Interest}

The authors declare that there are no conflicts of interest regarding the publication of this paper.

\section{Acknowledgments}

This work was supported by the National Key Research and Development Program of China (no. 2019YFB1705402) and National Natural Science Foundation of The People's Republic of China (Grant 61973041).

\section{References}

[1] Y. Sun and J. Yu, "Fault detection of rolling bearing using sparse representation-based adjacent signal difference," IEEE Transactions on Instrumentation and Measurement, vol. 70, pp. 1-16, 2021.
[2] Z. Liao, X. Song, B. Jia, and P. Chen, "Bearing fault feature enhancement and diagnosis based on statistical filtering and 1.5-dimensional symmetric difference analytic energy spectrum," IEEE Sensors Journal, vol. 21, no. 8, pp. 9959-9968, 2021.

[3] J. Ma and J. Xu, "Fault prediction algorithm for multiple mode process based on reconstruction technique," Mathematical Problems in Engineering, vol. 2015, Article ID 348729, 8 pages, 2015.

[4] N. E. Huang, Z. Shen, S. R. Long, and M. C. Wu, "The empirical mode decomposition and the Hilbert spectrum for nonlinear and non-stationary time series analysis," Proceedings of the Royal Society A: Mathematical, Physical and Engineering Sciences, vol. 454, pp. 903-995, 1998.

[5] N. E. Huang, M.-L. C. Wu, and S. R. Long, "A confidence limit for the empirical mode decomposition and Hilbert spectral analysis," Proceedings of the Royal Society A: Mathematical, Physical and Engineering Sciences, vol. 459, no. 2037, pp. 2317-2345, 2003.

[6] R. Jegadeeshwaran, V. Sugumaran, and K. P. Soman, "Vibration based fault diagnosis of a hydraulic brake system using variational mode decomposition (VMD)," Structural Durability \& Health Monitoring, vol. 10, pp. 81-97, 2014.

[7] H. Li, Z. Li, and W. Mo, "A time varying filter approach for empirical mode decomposition," Journal of Signal Processing, vol. 138, pp. 146-158, 2017.

[8] X. P. Ren, P. Li, and C. G. Wang, "Early fault diagnosis of rolling bearing based on VMD and fast spectral kurtosis," Bearings, vol. 12, pp. 39-43, 2017.

[9] A. Moshrefzadeh, A. Fasana, and J. Antoni, “The autogram:an effective approach for selecting the optimal demodulation band in rolling element bearing diagnosis," Mechanical Systems and Signal Processing, vol. 105, pp. 294-318, 2018.

[10] H. M. Ozaktas and D. Mendlovic, "Fractional Fourier transforms and their optical implementation II," Journal of the Optical Society of America a-option Image Science and Vision, vol. 10, no. 12, pp. 2522-2531, 1993.

[11] L. Jiao and J. Ma, "Feature extraction of rolling bearing faults based on VMD and FRFT," in Proceedings of the 2020 IEEE 9th Data Driven Control and Learning Systems Conference, DDCLS, pp. 167-172, Liuzhou, China, June 2020.

[12] J. J. Jia and Y. J. Huang, "Fault diagnosis of variable speed rolling bearing based on FRFT filter and LMS noise reduction," Journal of Beijing University of Chemical Technology, vol. 48, pp. 84-91, 2021.

[13] X. Zhang, Y. Liang, J. Zhou, and Y. Zang, "A novel bearing fault diagnosis model integrated permutation entropy, ensemble empirical mode decomposition and optimized SVM," Measurement, vol. 69, pp. 164-179, 2015.

[14] U. Jung and B. H. Koh, "Wavelet energy-based visualization and classification of high-dimensional signal for bearing fault detection," Knowledge and Information Systems, vol. 44, no. 1, pp. 197-215, 2015.

[15] A. Elen and E. Avuçlu, "Standardized variable distances: a distance-based machine learning method," Applied Soft Computing Journal.vol. 98, Article ID 106855, 2021.

[16] C. Ma, S. H. Wang, and X. L Xu, "Intelligent fault diagnosis method of rolling bearing based on CSBP," Journal of Electronic Measurement and Instrument, vol. 33, pp. 58-63, 2019.

[17] W. Zhang, G. L. Peng, C. H. Li, Y. Chen, and Z. Zhang, "A new deep learning model for fault diagnosis with good anti-noise and domain adaptation ability on raw vibration signals," Sensors, vol. 17, no. 2, pp. 387-425, 2017. 
[18] E. Uğur, "A precise and stable machine learning algorithm: eigenvalue classification (EigenClass)," Neural Computing and Applications, vol. 33, pp. 5381-5392, 2021.

[19] C. Ma, S. H. Wang, and X. L. Xu, "Fault diagnosis of acoustic array rolling bearing based on EEMD," Journal of Electronic Measurement and Instrument, vol. 31, pp. 1379-1384, 2017.

[20] K. D. Wang and K. K. Han, "Bearing fault diagnosis based on time varying filtering empirical mode decomposition," Manufacturing Technology \& Machine Tool, vol. 12, pp. 42-46, 2018.

[21] Y. G. Lei, Z. J. He, and Y. Y. Zi, “A new approach to intelligent fault diagnosis of rotating machinery," Expert Systems with Applications, vol. 35, no. 4, pp. 1593-1600, 2008. 